\title{
A new meta-heuristic for economic dispatch of integrated energy system with multi-type energy storages
}

\author{
Tao Liang ${ }^{1 *}$, Xiaodong Yin ${ }^{1}$ and Qun $\mathrm{Ge}^{1}$ \\ ${ }^{1}$ Shandong Electric Power Engineering Consulting Institute Corp., Ltd., 106 Minziqian Road Jinan, China
}

\begin{abstract}
Dispatch optimization of the integrated energy system with multi-type energy storages, as an essential issue to optimum energy management, is a high-dimensional complex nonlinear problem with timeseries constraints. To overcome the problem, a new meta-heuristic method is presented in this paper. The proposed method introduces several heuristic rules, called as maximum storage utilization heuristic rules, and embeds them into an adaptive GA to improve the quality of initial feasible solutions and new solutions in each iteration. In contrast to existing methods, the new method guarantees the convergence to an optimal solution with low computational cost. Simulation results indicate that the proposed method provides excellent performance in solving economic dispatch of integrated energy system with multi-type energy storages.
\end{abstract}

\section{Introduction}

Nowadays, the growing concerns about the energy crisis and environmental problems lead to changes of energy production and consumption patterns. The integrated energy system, as a stable and economical form of energy treatment, has attracted widespread attention owning to its high energy efficiency, low emission, reliability and wide applicability ${ }^{[1]}$.

Many studies have been done to solve the optimal dispatch for the integrated energy system, which is one of the most essential and principal issues to guarantee that the system works stably and efficiently. Several methods, such as linear programming (LP) ${ }^{[2]}$, mixed-integer programming (MIP) ${ }^{[3]}$, and mixed-integer non-linear programming (MINLP) ${ }^{[4]}$, have been applied to minimize the total cost or emission with operation constraints. However, the traditional methods are inadequate due to the high-dimensional, nonlinear, non-convex characters of the dispatch optimization problems, especially when the energy storage devices are used for peak load shifting in the integrated energy system. Currently, several metaheuristic based optimization approaches, such as genetic algorithm $(\mathrm{GA})^{[5]}$, particle swarm optimization $(\mathrm{PSO})^{[6]}$, artificial bee colony $(\mathrm{ABC})^{[7]}$, and harmony search $(\mathrm{HS})^{[8]}$ have been effectively applied to solve dispatch problems. Nevertheless, most of the existing researches only involve a single type of storage device, the dispatch optimization problems related to multiple types of storage are rarely mentioned.

In this paper, a new meta-heuristic method based on heuristic rules and GA is proposed for economic dispatch of integrated energy system with multi-type energy storages. Considering it is exceedingly difficult for the random search method to obtain a feasible solution, owing to the increasing complexity and the time-series correlation of variables in dispatch problems with multiple types of storage, several heuristic rules on optimal operation of energy storges are embedded into the GA to improve the quality of initial feasible solutions and new solutions in each iteration, which has better performance in searching for the feasible and optimal solutions. Therefore, the proposed method can be used to obtain the optimal scheduling strategy for the integrated energy system with multi-type energy storages. Some numerical simulation results are given to verify its excellent performance in optimal solutions as well as the calculating time.

\section{Problem formulation}

The integrated energy system with multi-type energy storages consist of power-supply components, heatsupply components, cool-supply components, and multitype energy storage systems. Typically, it includes photovoltaic (PV) systems, battery systems, electrical boilers (EB), air-source heat pumps (HP), water-source heat pumps water chillers and water tanks for heat or cool storage, as well as the grid. The dispatch optimization problem can be described as follows:

The goal of optimization is to minimize the total operation cost during the scheduling timespan within security constraints. The objective function can be written as:

$$
\begin{aligned}
& \min f=\sum_{t=1}^{T}\left\{p_{t}^{e b} Q_{t}^{e b}-p_{t}^{e s} Q_{t}^{e s}+\right. \\
& \left.\sum_{i=1}^{N}\left[\omega_{i}^{s t} X_{i t}\left(1-X_{i, t-1}\right)+\omega_{i}^{\text {om }} Q_{i t}^{\text {out }}\right]\right\}
\end{aligned}
$$

where $\mathrm{T}$ is the total dispatch period, $\mathrm{N}$ is the quantity of units that consume start-up and shutdown costs and maintenance costs. $p_{t}^{\text {eb }}$, $p_{t}^{\text {es }}$ are the prices of buy from and feed into the grid at time interval t. $Q_{t}^{\mathrm{eb}}, Q_{t}^{\mathrm{es}}$ are the

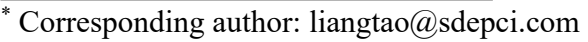


power of buy from and feed into the grid. $\omega_{i}^{\text {st }}$ is the specific cost of unit $i$ for once start-up or shutdown. $X_{i t}$ is a binary variable indicating whether the unit $\mathrm{i}$ is working at the time interval t. $\omega_{i}^{\text {om }}$ is the maintenance cost factor related to working capacity $Q_{i t}^{\text {out }}$.

The operational optimization should meet the constraints of energy balance, equipment capacity limits, grid power limits and load response limits. The constraints can be expressed as:

$$
\begin{gathered}
P_{t}^{e b}+\sum_{i \in M_{e o}} P_{i t}^{\text {out }}+\sum_{i \in M_{e s}} P_{i t}^{\text {out }}=\sum_{i \in M_{e i}} P_{i t}^{\text {in }}+ \\
\sum_{i \in M_{e s}} P_{i t}^{\text {in }}+P_{t}^{\text {esup }}+P_{t}^{\text {eloss }} \\
\sum_{i \in M_{c o}} P_{i t}^{\text {out }}+\sum_{i \in M_{c s}} P_{i t}^{\text {out }}=\sum_{i \in M_{c s}} P_{i t}^{\text {in }}+ \\
P_{t}^{\text {csup }}+P_{t}^{\text {closs }} \\
\sum_{i \in M_{h o}} P_{i t}^{\text {out }}+\sum_{i \in M_{h s}} P_{i t}^{\text {out }}=\sum_{i \in M_{h s}} P_{i t}^{\text {in }}+ \\
P_{t}^{\text {hsup }}+P_{t}^{\text {hloss }} \\
X_{i t} P_{i, \text { min }}^{\text {out }} \leq P_{i t}^{\text {out }} \leq X_{i t} P_{i, \text { max }}^{\text {out }} \\
\forall i \in M_{\text {co }} \cup M_{h o} \cup M_{e o} \\
X_{i t}^{\text {out }} P_{i, \text { min }}^{\text {out }} \leq P_{i t}^{\text {out }} \leq X_{i t}^{\text {out }} P_{i, \text { max }}^{\text {out }} \text { and } \\
X_{i t}^{\text {in }} P_{i, \text { min }}^{\text {in }} \leq P_{i t}^{\text {in }} \leq X_{i t}^{\text {in }} P_{i, \text { max }}^{\text {in }} \\
X_{i t}^{\text {out }}+X_{i t}^{\text {in }} \leq 1 \\
\forall i \in M_{c s} \cup M_{h s} \cup M_{e s} \\
0 \leq P_{t}^{\text {eb }} \leq P_{\text {max }}^{\text {eb }} \\
0 \leq P_{t}^{\text {es }} \leq P_{\text {max }}^{\text {es }} \\
P_{t}^{\text {creq }} \leq P_{t}^{\text {csup }} \\
P_{t}^{\text {hreq }} \leq P_{t}^{\text {hsup }} \\
P_{t}^{\text {ereq }}=P_{t}^{\text {esup }} \\
\end{gathered}
$$

where $\mathrm{M}_{\mathrm{co}}, \mathrm{M}_{\mathrm{ho}}, \mathrm{M}_{\mathrm{eo}}$ and $\mathrm{M}_{\mathrm{ei}}$ are the sets of cooling equipment, heat production equipment, power generation equipment and power consumption equipment, respectively. $\mathrm{M}_{\mathrm{cs}}, \mathrm{M}_{\mathrm{hs}}$ and $\mathrm{M}_{\mathrm{es}}$ are the sets of cooling storage equipment, heat storage equipment and electric storage equipment, respectively. $\mathrm{P}_{i, \text { min }}^{\text {out }}, \mathrm{P}_{\mathrm{i} \text {,max }}^{\text {out }}$ are the minimum and maximum power outputs of the unit $\mathrm{i}$. $\mathrm{X}_{\mathrm{it}}^{\mathrm{in}}$, $\mathrm{X}_{\mathrm{it}}^{\text {out }}$ are binary variables indicating whether the storage equipment $\mathrm{i}$ is storing or releasing energy at the time interval t. $\mathrm{P}_{\mathrm{i} \text {,min }}, \mathrm{P}_{\mathrm{i} \text {,max }}^{\mathrm{in}}$ are the minimum and maximum storing power of the storage equipment $\mathrm{i}$. $\mathrm{P}_{\max }^{\mathrm{es}}$ is the maximum power fed into the grid, while $\mathrm{P}_{\max }^{\mathrm{eb}}$ is the maximum power drawn from the grid. $\mathrm{P}_{\mathrm{t}}^{\text {creq }}, \mathrm{P}_{\mathrm{t}}^{\text {hreq }}$, and $\mathrm{P}_{\mathrm{t}}^{\mathrm{ereq}}$ represent the cold load, heat load and electricity load of users at the time interval $t$, while $\mathrm{P}_{t}^{\text {csup }}, \mathrm{P}_{\mathrm{t}}^{\text {hsup }}$, $\mathrm{P}_{\mathrm{t}}^{\text {esup }}$ represent the cold, heat, electricity energy supplied to the users at the time interval $t$, separately.

For the energy conversion devices in integrated energy systems, such as electrical boiler, heat pump, or water chiller, the relationship between energy cost and output is as below:

$$
P_{i t}^{o u t}=\eta_{i t} \cdot P_{i t}^{\text {in }}
$$

where $\eta_{\text {it }}$ is the efficiency of the unit $i$ at the time interval $\mathrm{t}$. For heat pump in particular, $\eta_{i t}$ represents the coefficient of performance.

The energy storage equipment, as a typical time-series component, needs to meet the following dynamic constraints:

$$
\begin{gathered}
S_{i t}=S_{i, t-\Delta t}\left(1-\mu_{i t}^{\text {loss }}\right)+ \\
P_{i t}^{\text {in }} \cdot \Delta t \cdot \eta_{i t}^{\text {in }}-P_{i t}^{\text {out }} \cdot \Delta t / \eta_{i t}^{\text {out }} \\
S_{i}^{\text {min }} \leq S_{i t} \leq S_{i}^{\text {max }}
\end{gathered}
$$

where $S_{i t}$ is the energy storage state of the storage equipment $i$ at the time interval $t$. $\mu_{\mathrm{it}}^{\text {loss }}$ is the loss rate of energy storage, and $\eta_{i t}^{\text {in }}, \eta_{i t}^{\text {out }}$ represent the storing efficiency and releasing efficiency. $S_{i}^{\min }, S_{i}^{\max }$ are the minimum and maximum storage capacity of energy storage equipment $i$, respectively. It is hard to solve the dispatch optimization problem with energy storages owing to the efficiency of the unit, such as $\eta_{i t}, \eta_{i t}^{\text {in }}, \eta_{i t}^{\text {out }}$, is not a fixed value or a linear function of the process parameters, such as temperature, flow, load rate, etc. during operation. Besides, the energy consumption of large auxiliary devices, for example, the hot net circulation pumps, needs to be considered due to it is sometimes as much as the cost of energy conversion devices.

\section{Improved genetic algorithm}

The GA has been applied popularly as a useful optimization tool for handling optimal dispatch problems. However, with the increasing number and complexity of components in the integrated energy system, the GA and its existing improved versions are flawed, which are usually exposed to premature convergence and high computational cost. Especially for the dispatch problems of integrated energy system with multi-type energy storages, these methods may fail to find a feasible solution due to the complex coupling time-series constraints. To solve the above problem, this paper presents a new approach by implanting several heuristic rules on optimal operation of energy storges into an adaptive GA to improve the quality of initial feasible solutions and new solutions in each iteration, ensuring the delivery of the global optimal solution with low computational cost.

\subsection{Maximum storage utilization heuristic rules}

For the integrated energy system with energy storages, it is a recognized effective economy measure that maximum application of energy storage devices to shift peak load and reduce the cost of purchased electricity in accordance with peak-valley time-of-use power price. Hence, maximum storage utilization heuristic rules are proposed to determine the optimal storing and releasing power of energy storages for each store-and-release cycle. The heuristic rules are given below:

(A) Store-and-release cycles determining 
The number of store-and-release cycles is equal to the number of peak-valley cycles of time-of-use power price in the scheduling timespan.

(B) Upper and lower releasing power limits

For cold, heat or electric storage devices, the upper and lower releasing power limits at a given time interval can be calculated as:

$$
\begin{gathered}
P_{t}^{O H}=\min \left(P_{\max }^{o}, P_{t}^{r e q}\right) \\
P_{t}^{O L}=\max \left(0, P_{t}^{r e q}-\hat{P}_{\max }^{o}\right)
\end{gathered}
$$

where $P_{t}^{r e q}$ is the corresponding energy load of users at the time interval $t, P_{\text {max }}^{o}$ is the maximum releasing of the energy storage, $\hat{P}_{\max }^{o}$ is the sum of maximum power outputs of corresponding energy supply devices except the storages.

(C) Maximum economic energy storage

The economic energy storage in a given store-andrelease cycle can be expressed as:

$\hat{S}^{\max }=$

$$
\begin{gathered}
\min \left(S^{\max }, \sum_{t \in \widehat{T}_{v}} P_{\max }^{I} \Delta t, \sum_{t \in T_{p} \cup T_{l}} P_{t}^{O H} \Delta t+\right. \\
\left.\sum_{t \in T_{v}} P_{t}^{O L} \Delta t\right)
\end{gathered}
$$

where $S^{\max }$ is the maximum storage capacity of the energy storage, $P_{\max }^{I}$ is the maximum storing power of the energy storage, $T_{p}, T_{l}$ and $T_{v}$ are the sets of time intervals in the peak, flat and valley segments according to time-ofuse power price, respectively. $\widehat{T}_{v}$ is the set of time intervals in the valley segment and satisfying $P_{t}^{O L}=0$.

(D) Optimal storing and releasing power determining

The optimal storing and releasing power of energy storage can be calculated as:

For the time interval $t$ in the peak segment $t \in T_{p}$ :

$$
P_{t}^{O}=
$$

$$
\left\{\begin{array}{cl}
P_{t}^{O H}-\frac{\Delta P_{1} \cdot\left(P_{t}^{O H}-P_{t}^{O L}\right)}{\sum_{t \in T_{p}\left(P_{t}^{O H}-P_{t}^{O L}\right)}} & \hat{S}^{\max }<\sum_{t \in T_{p}} P_{t}^{O H} \Delta t+\sum_{t \in T_{l}+T_{v}} P_{t}^{O L} \Delta t \\
P_{t}^{O H} & \hat{S}^{\text {max }} \geq \sum_{t \in T_{p}} P_{t}^{O H} \Delta t+\sum_{t \in T_{l}+T_{v}} P_{t}^{O L} \Delta t
\end{array}\right.
$$

For the time interval $\mathrm{t}$ in the flat segment $t \in T_{l}$ : $P_{t}^{O}=$

$$
\left\{\begin{array}{cl}
P_{t}^{O L} & \hat{S}^{\max } \leq \sum_{t \in T_{p}} P_{t}^{O H} \Delta t+\sum_{t \in T_{l}+T_{v}} P_{t}^{O L} \Delta t \\
P_{t}^{O L}+\frac{\Delta P_{2} \cdot\left(P_{t}^{O H}-P_{t}^{O L}\right)}{\sum_{t \in T_{l}\left(P_{t}^{O H}-P_{t}^{O L}\right)}} & \hat{S}^{\text {max }}>\sum_{t \in T_{p}} P_{t}^{O H} \Delta t+\sum_{t \in T_{l}+T_{v}} P_{t}^{O L} \Delta t
\end{array}\right.
$$

For the time interval $\mathrm{t}$ in the valley segment $t \in T_{v}$ :

$$
P_{t}^{O}=\left\{\begin{array}{cl}
P_{t}^{O L} & P_{t}^{O L}>0 \\
(-1) \cdot\left(P_{\max }^{I}-\frac{\Delta P_{3} \cdot P_{\max }^{I}}{\sum_{t \in T_{v}-\bar{T}_{v}} P_{\max }^{I}}\right) & P_{t}^{O L}=0
\end{array}\right.
$$

Where $P_{t}^{O}$ is storing and releasing power of the given energy storage, and positive values represent releasing, while negative values represent storing. $\Delta P_{1}, \Delta P_{2}$ and $\Delta P_{3}$ are intermediate variables:

$$
\begin{gathered}
\Delta P_{1}=\left(\sum_{t \in T_{p}} P_{t}^{O H} \Delta t+\sum_{t \in T_{l}+T_{v}} P_{t}^{O L} \Delta t-\hat{S}^{\text {max }}\right) / \Delta t \\
\Delta P_{2}=\left(\hat{S}^{\text {max }}-\sum_{t \in T_{p}} P_{t}^{O H} \Delta t-\sum_{t \in T_{l}+T_{v}} P_{t}^{O L} \Delta t\right) / \Delta t \\
\Delta P_{3}=\left(\sum_{t \in T_{v}-\bar{T}_{v}} P_{\text {max }}^{I} \Delta t-\hat{S}^{\text {max }}\right) / \Delta t \\
\text { (E) Dispatch plan generation }
\end{gathered}
$$

With the maximum storage utilization heuristic rules, an optimized operating strategy of energy storages is produced. And then, the economic dispatch plan can be generated by load distribution to the other energy conversion devices in sequence from high to low energy efficiency, following the principle of energy supply and demand balance.

\subsection{Main steps of the proposed algorithm}

The main steps of the proposed improved genetic algorithm can be summarized as follows:

Step 1: Initialize the algorithm parameters and genetic encoding.

Set the iteration $l=1$, population size $M$, number of iterations $N$ and convergence precision $\epsilon$. Each individual should consist of all the decision variables, including the start-stop status and output power of each equipment at each time, the energy store-release status, storing power and releasing power of each energy storage device, according to the economic dispatch model of the integrated energy system.

Step 2: Generate the initial population.

Firstly, generate a feasible solution applying the maximum storage utilization heuristic rules. And then, one-third of the individuals are generated by random mutation of the above feasible solution, while the other individuals are generated randomly within the equipment capacity limits.

Step 3: Evaluate the fitness of each individual.

The fitness function adopts the sum of the objective function and the penalty term for constraint violation.

Step 4: Update population by selection, crossover, mutation.

The best individual in the current population is copied directly to the next population using an elite retention strategy. The crossover probability $p_{c}$ and mutation probability $p_{m}$ are dynamic adjusted with population diversity ${ }^{[9]}$ :

$$
\begin{gathered}
p_{c}=1 /\left[1+\exp \left(-k_{1} \Delta-c_{1}\right)\right] \\
p_{m}=1 /\left[1+\exp \left(-k_{2} \Delta-c_{2}\right)\right]
\end{gathered}
$$

where $k_{1}, k_{2}>0, c_{1}, c_{2}>0$ are the control parameters, $\Delta$ represents the population diversity.

Step 5: Improve some individual quality by heuristic rules.

In this step, the maximum storage utilization heuristic rules are used to improve the quality of some new individuals in the new population, which can be selected with probability $p_{i} \in[0.05,0.25]$. Meanwhile, the parameter $\hat{S}^{\max }$ in Eq. (18) - (23) should be replaced with $\hat{S}_{\text {ind }}^{\max }, \hat{S}_{\text {ind }}^{\max }=\min \left(\hat{S}^{\max }, \sum_{t \in T_{v}} \tilde{P}_{t}^{i n} \Delta t\right)$, where $\tilde{P}_{t}^{i n}$ is the storing power of the energy storage at the time interval $t$ in the dispatch plan corresponding to the selected individual.

Step 6: Check stopping criteria and repeat steps 3-5.

The optimization process is repeated from step 3 to step 5 until the termination criteria are satisfied.

\section{Results and discussion}

This section applies a practical integrated energy system to verify the efficiency and effectiveness of the proposed algorithm. The integrated energy system whose composition is shown in Table 1, provides electric power, cooling, and heating energy for buildings. The economic dispatch problem selected a natural day as an optimized cycle, and the peak-valley time-of-use power price was considered, as well as the equipment efficiency variation 
during operation. Thus, this case is a large-scale nonlinear mixed integer planning problem.

Table 1. Parameters of the integrated energy system.

\begin{tabular}{|c|c|c|}
\hline Facility & Quantity & Unit capacity \\
\hline PV & $1 \mathrm{set}$ & $650 \mathrm{kWp}$ \\
\hline Water chillers & 3 & $2110 \mathrm{~kW} / 2110 \mathrm{~kW} / 1040 \mathrm{~kW}$ \\
\hline Electrical boilers & 2 & $630 \mathrm{~kW}$ \\
\hline Air-source heat pumps & 22 & $106 \mathrm{~kW}$ \\
\hline $\begin{array}{c}\text { Water-source heat } \\
\text { pumps }\end{array}$ & 1 & $81 \mathrm{~kW}$ \\
\hline $\begin{array}{c}\text { Water tanks for heat } \\
\text { or cool storage }\end{array}$ & 1 & $\begin{array}{c}\text { Cool storage: } 9280 \mathrm{kWh} \\
\text { Heat storage: } 17280 \mathrm{kWh}\end{array}$ \\
\hline Battery systems & 2 & $410 \mathrm{kWh} / 615 \mathrm{kWh}$ \\
\hline $\begin{array}{c}\text { Hot net circulation } \\
\text { pumps }\end{array}$ & 3 & $380 \mathrm{t} / \mathrm{h}, 55 \mathrm{~kW}$ \\
\hline
\end{tabular}

The proposed improved genetic algorithm was implemented in $\mathrm{C}++$ language on a $\mathrm{PC}$ with $2.4 \mathrm{GHz} \mathrm{CPU}$ and $8 \mathrm{G}$ RAM. To manifest the performance of the proposed algorithm, the case has been tested and compared with the results obtained by traditional adaptive GA without heuristic rules for the different parameters values, and computational results are summarized in Table 2. The maximum number of iterations $N$ is fixed as 10000 . While the population size is set as 100,200 and 500 to make a comparison.

For six times independent calculations, the optimization results obtained using the proposed method are all equal to 10484.68 with $M=200$, while five times equal to 10493.75 with $M=100$. However, there are random fluctuations in the results obtained using the traditional adaptive GA method, in which the best result is 15418.20 and the worst result is 15741.21 , with $M=200$ for example. Although the calculating time of the traditional adaptive GA method is a little shorter than the proposed method with the same parameter values, the results should not be adopted because the objectives of operation cost exceeds $150 \%$ of the optimized value. Compared with the traditional adaptive GA with $M=500$, the proposed method with $M=200$ does have advantages both in the optimal solution and in the calculating time. The convergence procedures of various algorithms are depicted in Figure 1. Thanks to the application of heuristic rules, the approximate solution will be quickly found, and can be converged to the optimal solution at a faster rate.

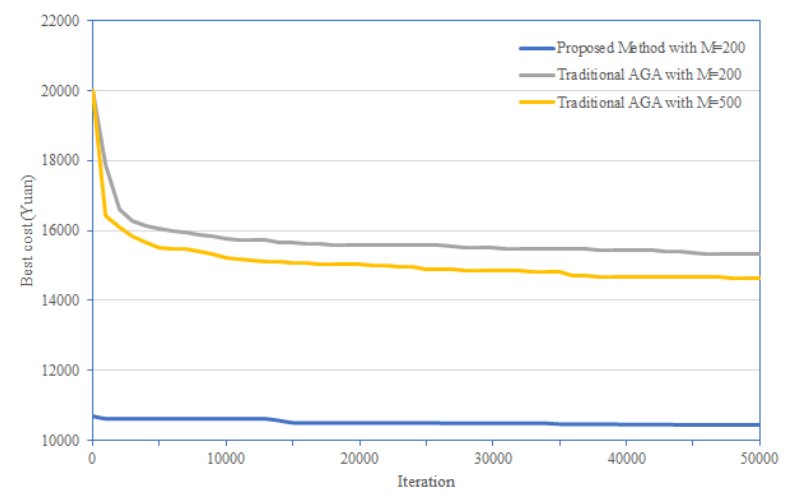

Figure 1. The convergence procedures of various algorithms.

\section{Conclusion}

In this paper, a new meta-heuristic method based on heuristic rules and GA is proposed to solve economic

Table 2 Objective function values and calculating times of various algorithms.

\begin{tabular}{|c|c|c|c|c|c|c|c|c|c|}
\hline Method & Parameters & Results & $1 s t$ & $2 n d$ & $3 r d$ & $4 t h$ & 5 th & 6th & Average \\
\hline \multirow{2}{*}{$\begin{array}{l}\text { Proposed } \\
\text { algrithm }\end{array}$} & $M=100$ & $\begin{array}{l}\text { Objective (yuan) } \\
\text { Calculating time(s) }\end{array}$ & $\begin{array}{l}10493.75 \\
43.58\end{array}$ & $\begin{array}{l}10493.75 \\
41.33\end{array}$ & $\begin{array}{l}10503.79 \\
44.42\end{array}$ & $\begin{array}{l}10493.75 \\
45.05\end{array}$ & $\begin{array}{l}10493.75 \\
43.48\end{array}$ & $\begin{array}{l}10493.75 \\
42.64\end{array}$ & \begin{tabular}{|l|}
10495.42 \\
43.42
\end{tabular} \\
\hline & $M=200$ & $\begin{array}{l}\text { Objective (yuan) } \\
\text { Calculating time(s) }\end{array}$ & $\begin{array}{l}10484.68 \\
81.22 \\
\end{array}$ & $\begin{array}{l}10484.68 \\
82.11 \\
\end{array}$ & $\begin{array}{l}10484.68 \\
83.59\end{array}$ & $\begin{array}{l}10484.68 \\
80.78\end{array}$ & $\begin{array}{l}10484.68 \\
82.01\end{array}$ & $\begin{array}{l}10484.68 \\
81.57 \\
\end{array}$ & $\begin{array}{l}10484.68 \\
81.88\end{array}$ \\
\hline \multirow{3}{*}{$\begin{array}{c}\text { Traditional } \\
\text { adaptive } \\
\text { GA }\end{array}$} & $M=100$ & $\begin{array}{l}\text { Objective (yuan) } \\
\text { Calculating time(s) }\end{array}$ & $\begin{array}{l}15741.21 \\
33.22 \\
\end{array}$ & $\begin{array}{l}16153.69 \\
31.27 \\
\end{array}$ & $\begin{array}{l}15865.89 \\
32.38 \\
\end{array}$ & $\begin{array}{l}16200.63 \\
34.42 \\
\end{array}$ & $\begin{array}{l}15891.04 \\
31.33 \\
\end{array}$ & $\begin{array}{l}15835.74 \\
32.59 \\
\end{array}$ & $\begin{array}{l}15948.03 \\
32.54 \\
\end{array}$ \\
\hline & $M=200$ & $\begin{array}{l}\text { Objective (yuan) } \\
\text { Calculating time(s) }\end{array}$ & $\begin{array}{l}15611.24 \\
63.36\end{array}$ & $\begin{array}{l}15705.85 \\
68.73 \\
\end{array}$ & $\begin{array}{l}15582.45 \\
64.2 \\
\end{array}$ & $\begin{array}{l}15418.20 \\
59.72\end{array}$ & $\begin{array}{l}15595.87 \\
65.72 \\
\end{array}$ & $\begin{array}{l}15741.21 \\
67.59 \\
\end{array}$ & $\begin{array}{l}15609.14 \\
64.89\end{array}$ \\
\hline & $M=500$ & $\begin{array}{l}\text { Objective (yuan) } \\
\text { Calculating time(s) }\end{array}$ & $\begin{array}{l}15258.57 \\
152.25\end{array}$ & \begin{tabular}{|l|}
15319.3 \\
153.52
\end{tabular} & \begin{tabular}{|l|}
15399.29 \\
153.87
\end{tabular} & $\begin{array}{l}14850.11 \\
152.34\end{array}$ & $\begin{array}{l}15284.93 \\
156.26\end{array}$ & $\begin{array}{l}15457.26 \\
153.12\end{array}$ & $\begin{array}{l}15261.58 \\
153.56\end{array}$ \\
\hline
\end{tabular}

dispatch problems of integrated energy system with multitype energy storages. To deal with the increasing complexity and the time-series correlation of variables in dispatch problems with multiple types of storage, the maximum storage utilization heuristic rules are presented and embedded into the adaptive GA to enhance the optimization performances. Simulation results and comparisons with the traditional adaptive GA method based on a practical integrated energy system demonstrate the effectiveness, efficiency of the proposed algorithm. Future work will focus on the optimal real-time dispatch and control of integrated energy system with multi-type energy storages.

\section{References}

1. W. Gu, J. Wang, S. Lu, et al. Optimal operation for integrated energy system considering thermal inertia of district heating network and buildings, Applied Energy, 2017, 199: 234-246.

2. M.K. Bai, Y. Wang, W. Tang, et al. Day-ahead optimal dispatching of regional integrated energy 
system based on interval linear programming, Power System Technology, 2017, 41: 3963-3970.

3. Y. Wang, N. Zhang, Z.Y. Zhuo, et al. Mixed-integer linear programming-based optimal configuration planning for energy hub: Starting from scratch, Applied Energy, 2018, 210: 1141-1150.

4. N Deng, R Cai, Y Gao, et al. A MINLP model of optimal scheduling for a district heating and cooling system: a case study of an energy station in Tianjin. Energy, 2017, 141: 1750-1763.

5. R.M. Fang, Multi-objective optimized operation of integrated energy system with hydrogen storage, Int. J. Hydrogen Energy, 2019, 44: 29409-29417.

6. Z.J. Bao, D.W. Chen, L. Wu, et al. Optimal inter-and intra-hour scheduling of islanded integrated-energy system considering linepack of gas pipelines, Energy, 2019, 171: 326-340.

7. S. Sharifi, M. Sedaghat, P. Farhadi, et al. Environmental economic dispatch using improved artificial bee colony algorithm, Evolving Systems, 2017, 8: 233-242.

8. M.l. Wang, T Zhang, P.h. Wang, et al. An improved harmony search algorithm for solving day-ahead dispatch optimization problems of integrated energy systems considering time-series constraints, Energy \& Buildings, 2020, 229: 110477.

9. J. Zou. Adaptive genetic algorithm successive approximation and its application to long-term resources operation, System Engineering Theory \& Practice, 2013, 33, 267-272. 